\title{
Venous Invasion in Colorectal Cancer: Impact of Morphologic Findings on Detection Rate
}

\section{Chungsu Hwang, MD \\ Sojeong Lee, MD \\ Ahrong Kim, MD \\ Young-Geum Kim, MD \\ Sang-Jeong Ahn, MD \\ Do Youn Park, MD, PhD}

Department of Pathology, Biomedical Research Institute,

Pusan National University Hospital,

Pusan National University

School of Medicine, Busan, Korea
Correspondence: Do Youn Park, MD, PhD

Department of Pathology,

Biomedical Research Institute,

Pusan National University Hospital,

Pusan National University School of Medicine,

179 Gudeok-ro, Seo-gu, Busan 49241, Korea

Tel: 82-51-240-7717

Fax: 82-52-256-0788

E-mail: pdy220@pusan.ac.kr

\begin{abstract}
Purpose
Venous invasion (VI) is widely accepted as a poor prognostic factor in colorectal cancer (CRC), and is indicated as a high-risk factor determining the use of adjuvant chemotherapy in CRC. However, there is marked interobserver and intraobserver variability in VI identification and marked variability in the real prevalence of VI in CRC.
\end{abstract}

\section{Materials and Methods}

We investigated the detection rate of $\mathrm{VI}$ in 93 consecutive cases of T3 or T4 CRC based on the following: original pathology report, review of hematoxylin and eosin (H\&E) slides with attention to the "protruding tongue" and "orphan arteriole" signs, and elastic stain as the gold standard.

\section{Results}

Overall, the detection rate of VI was significantly increased as follows: 14/93 (15.1\%) in the original pathology report, 38/93 (40.9\%) in review of H\&E slides with attention to the "protruding tongue" and "orphan arteriole" signs, and 45/93 (48.4\%) using elastic stain. VI detection based on morphologic features showed $77.8 \%$ sensitivity and $91.1 \%$ specificity and showed a linear correlation (Spearman correlation coefficient, $0.727 ; p<0.001$ ) with $\mathrm{VI}$ detected by elastic stain. In addition, improved agreement between detection methods (detection on the basis of morphologic features, $\mathrm{k}=0.719 \mathrm{vs}$. original pathology report, $\mathrm{K}=0.318$ ) was observed using kappa statistics.

\section{Conclusion}

Slide review with special attention to the "protruding tongue" and "orphan arteriole" signs could be used for better identification of VI in CRC in routine surgical practice.
Key words

Colon, Rectum, Adenocarcinoma, Vein, Elastin

\section{Introduction}

Colorectal cancers (CRCs) are one of the most important causes of cancer-related death worldwide and have recently shown an increased incidence in Korea. Meticulous handling of resected specimens with accurate pathologic assessment is of particular importance in CRC patients [1,2]. Venous invasion (VI), or "large vessel" invasion, is a well-known independent prognostic factor of CRCs [3-5]. The VI status should be included in the surgical pathology report for CRC patients, according to the protocols of the College of American Pathologists (CAP) [1] and the Korean standardized pathology report for CRCs [2]. Furthermore, detection of VI in stage II CRC suggests the need for prompt adjuvant chemotherapy by oncologists [6]. Nevertheless, there is marked variability in the detection rate of VI, and there are no consensus guidelines regarding detection methods for VI in CRC. The prevalence of VI is widely distributed and influenced by various factors, including the experience or 
subspecialty of the pathologists, diagnostic criteria, numbers of blocks, and use of special stains [7-10]. In a cross-sectional study of 198 pathologists in Ontario, Canada, the majority of pathologists $(70.2 \%)$ reported a VI detection rate of less than $10 \%$ [11]. Population-based data from two cancer registries showed a VI detection rate in CRC of only $10 \%-18 \%[12,13]$. Regarding the detection rate of VI in CRC, the experience of pathologists as a subspecialty and the use of specific morphologic findings and elastic stain for detection of VI are important factors. Kirsch et al. [14] reported that gastrointestinal (GI) specialist pathologists detected more VI than non-GI pathologists ( $30.0 \%$ vs. $9.2 \%$ ), and that the use of elastic stain increased the VI detection rate compared to routine hematoxylin and eosin (H\&E) stain $(46.4 \%$ vs. $19.6 \%)$. Collectively, these previous reports reinforce that $\mathrm{VI}$ is an independent prognostic factor for CRCs and that pathologists should pay attention to its detection during the routine surgical handling of resected CRC specimens.

However, the use of elastic stain entails additional efforts and costs, including selection of tissue blocks for elastic stain after routine grossing of the specimen with delay of the turnaround time for final diagnosis, as well as additional costs. Recently, characteristic morphologic features (protruding tongue and orphan arteriole signs) implying VI were suggested as a method for detection of VI [14,15]. With this background information, we aimed to evaluate whether any morphologic findings providing clues to VI could correspond to VI detected by elastic stain, through the retrospective review of a series of 93 cases of T3 or T4 CRC.
We analyzed extramural VI (VI beyond muscularis propria) with prognostic significance $[1,6]$. VI was assessed in the 93 included cases of CRC in three steps: (1) evaluation of the original surgical pathology report, (2) re-evaluation of all H\&E-stained slides by two pathologists with close attention to morphologic features implying VI as described by Kirsch et al. [14], and (3) re-evaluation of equivocal features based on H\&E slides using elastic stains. Regarding morphologic features implying VI, we evaluated the protruding tongue sign (smooth oval-shaped protrusion of tumor cells into periserosal fat tissue near an artery) and the orphan arteriole sign (round tumor nodule near an artery without an accompanying vein) (Fig. 1). For elastic stain, elastic staining was performed using a Roche Elastic Staining Kit (860-005, Ventana Medical Systems Inc., Tucson, AZ) according to the manufacturer's instructions in all 93 cases of CRCs. One tissue block with equivocal features of VI on H\&E stained slides was selected.

The data were analyzed using the Student's $t$ test, Fisher exact test, or the chi-square test for differences between groups. The relationships between different detection methods were assessed using a Spearman rank correlation coefficient. Agreement between different detection methods was assessed with a kappa $(\kappa)$ value statistics. A value of $p<0.05$ was considered statistically significant. Statistical calculations were performed using SSPS ver. 21.0 (IBM Co., Armonk, NY).

\section{Results}

\section{Materials and Methods}

Ninety-three patients with T3 or T4 CRCs were selected among 132 patients who consecutively underwent resection of primary CRC at Pusan National University Hospital (PNUH) between January 2015 and May 2015. The group consisted of 52 men and 41 women with a mean age of 67.5 years (range, 31 to 87 years). This study was approved by the institutional review board (PNUH IRB 2011-16-2, revised at 2014-08-14). The following clinicopathological factors were assessed according to the Korean Standardized Pathology Report for Colorectal Cancer as well as the American Joint Committee on Cancer Staging Manual, seventh edition: age, tumor size, sex, location, histologic type, perineural invasion, lymphovascular emboli, lymph node metastasis, depth of invasion, and growth pattern. Numbers of tissue blocks and number of tissue blocks per tumor diameter $(\mathrm{cm})$ for the primary tumor mass were also evaluated.
Overall, the detection rate of VI was significantly increased as follows: $14 / 93$ in the original pathology report (15.1\%), $38 / 93$ in review of H\&E slides (40.9\%) with attention to the protruding tongue and orphan arteriole signs, and 45/93 (48.4\%) using elastic stain, respectively (Table 1). The tumor nodule with a protruding tongue or orphan arteriole sign was identified. When searching under H\&E stain, endothelial cells or the smooth muscular layer of a vessel around the tumor nodule were not identified. However, elastic staining showed the elastic layer of a vein around the tumor nodule with the protruding tongue or orphan arteriole signs (Figs. 2 and 3).

Detection of VI on the basis of morphologic features (protruding tongue and orphan arteriole) showed $77.8 \%$ sensitivity, $91.1 \%$ specificity, $92.1 \%$ positive predictive value, $81.8 \%$ negative predictive value, a positive likelihood ratio of 12.44 (95\% confidence interval [CI], 4.11 to 37.64$)$, and a negative likelihood ratio of 0.24 (95\% CI, 0.14 to 0.41 ). In addition, detection of VI with special attention to morpho- 

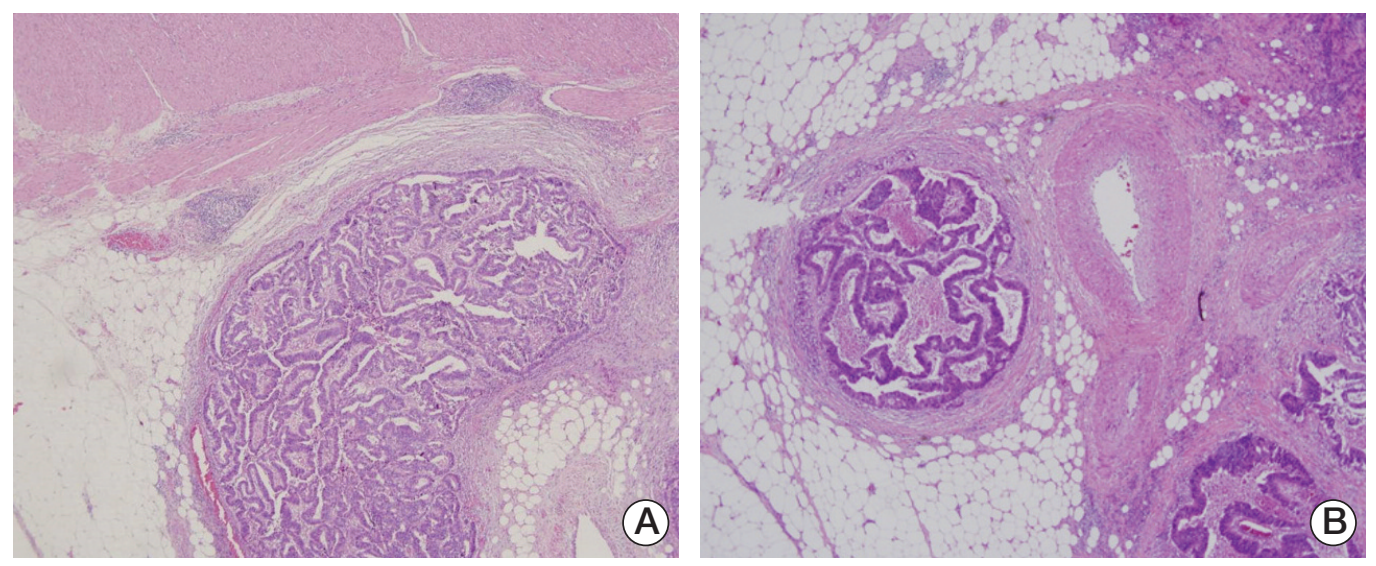

Fig. 1. (A) Protruding tongue sign. The oval tumor mass protruded beyond the tumor border into pericolic fat tissue (H\&E staining, $\times 40)$. (B) Orphan arteriole sign. Rounded tumor nodules adjacent to an artery without an accompanying vein were seen in the pericolic fat tissue (H\&E staining, $\times 40)$.

Table 1. Relationship between venous invasion detected by morphologic findings, original pathology report, and elastic stain in 93 cases of $\mathrm{T} 3$ or $\mathrm{T} 4$ colorectal cancer

\begin{tabular}{lccc} 
Venous invasion & No. & Elastic stain-negative $(\mathrm{n}=\mathbf{4 8})$ & Elastic stain-positive $(\mathrm{n}=\mathbf{4 5})$ \\
\hline Original report & & & \\
$\quad$ Negative & 79 & 48 & 14 \\
$\quad$ Positive & 14 & 0 & 10 \\
Morphologic finding & 55 & 45 & 35 \\
$\quad$ Negative & 38 & 3 & \\
\hline Positive & & & \\
\hline
\end{tabular}

logic features showed a linear correlation (Spearman correlation coefficient, $0.727 ; \mathrm{p}<0.001$ ) and with VI detected by elastic stain as the gold standard (Table 1). In comparison, detection of VI on the original pathology report showed only $33.1 \%$ sensitivity, $100.0 \%$ specificity, $100.0 \%$ positive predictive value, $60.8 \%$ negative predictive value, and a negative likelihood ratio of 0.69 (95\% CI, 0.57 to 0.84 ). Also, detection of VI with the original pathology report showed a linear correlation (Spearman correlation coefficient, 0.435; $\mathrm{p}<0.001$ ) with VI detected by elastic stain as the gold standard (Table 1). In addition, improved agreement was observed between detection methods (detection on the basis of morphologic features, $\kappa=0.719$ vs. original pathology report, $\kappa=0.318)$ using kappa statistics. These data indirectly suggest that morphologic findings (protruding tongue and orphan arteriole signs) are a better method of detection and could enhance the rate of detection of VI in CRC patients. The presence of VI identified by elastic stain was associated with lymph node metastasis, lymphatic emboli, and perineural invasion of tumor cells in T3 or T4 CRCs (Table 2).
In evaluation of the numbers of tissue blocks and number of tissue blocks / tumor diameter $(\mathrm{cm})$ for the primary tumor mass between VI-negative and VI-positive CRCs by elastic stain, there were no differences in the numbers of tissue blocks or number of tissue blocks/tumor diameter $(\mathrm{cm})$ (6.64 \pm 0.41 vs. $6.62 \pm 0.32, \mathrm{p}=0.964 ; 1.20 \pm 0.09$ vs. $1.31 \pm 0.07$, $\mathrm{p}=0.341)$.

\section{Discussion}

In this study, we demonstrate that the detection rate of VI was significantly increased in review of H\&E slides with attention to the "protruding tongue" and "orphan arteriole" signs as well as the use of elastic stain, and we recommend evaluation of these morphological findings for better identification of VI in CRCs in routine surgical practice. According to many reports, VI, or "large vessel" invasion, is an inde- 

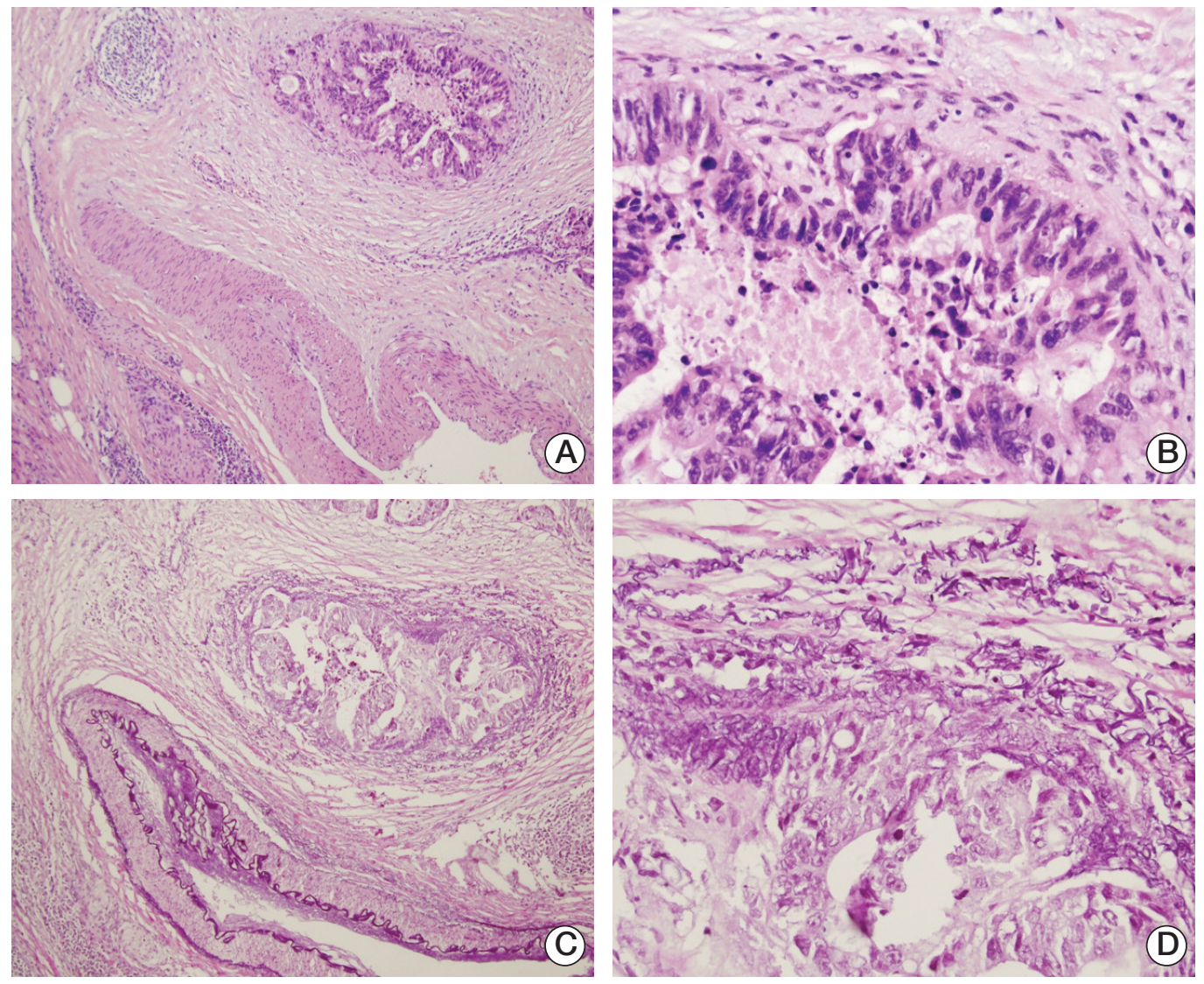

Fig. 2. (A) The tumor nodule with orphan arteriole sign was identified (H\&E staining, $\times 100)$. (B) At high power, endothelial cells of a vessel around the tumor nodule were not identified with some spindle cells (H\&E staining, $\times 400$ ). (C, D) Elastic staining showed the thick fragmented elastic layer of a vessel around the tumor nodule with the orphan arteriole sign $(\mathrm{C}$, elastic stain, $\times 100$; D, elastic stain, $\times 400)$.

pendent poor prognostic factor for CRCs [3-5], and detection of VI in stage II CRC suggests the need for prompt adjuvant chemotherapy by oncologists [6]. Therefore, the VI status should be included in the surgical pathology report of CRC patients by the various CRC reporting protocols, including those of the Royal College of Pathologists (RC Path, London, UK), the Royal College of Pathologists of Australia (RCPA), the CAP, the Japanese Society of Cancer of the Colon and Rectum, and the Korean Standardized Pathology Report for Colorectal Cancer [1,2,16-18].

There are two problems related to VI detection in routine surgical practice. The first is the marked variability in the detection rate of VI, and the other is the lack of consensus guidelines regarding detection methods for VI in CRC. In relation to the variability in the detection rate of VI, there are many contributing factors, including the experience or subspecialty of pathologists, diagnostic criteria, numbers of blocks, and usage of special stains [7-10]. Studies from
Ontario, Canada reported these problems and suggested ways to overcome these complicated issues $[11,14,15]$. Messenger et al. [11] reported that pathologists with a universityaffiliated center, a GI pathology subspecialist interest, and acceptance of the "orphan arteriole" sign were associated with a VI detection rate above 10\% [11]. In accordance with the above reports, our data showed an improved detection rate of VI with review of H\&E slides with specific attention to the "protruding tongue" and "orphan arteriole."

Kirsch et al. [14] reported that intraobserver agreement between GI and non-GI pathologists was improved with the use of elastic stain in VI detection in CRCs. Messenger et al. [19] and Dawson et al. [20] recommend routine elastic staining on all tumor blocks or on blocks that show the full thickness of the tumor. However, if elastic staining is ordered after slide review, additional costs and efforts are entailed, and turnaround times are delayed. If possible, it is recommended that elastic staining be requested at the time of grossing. Our 

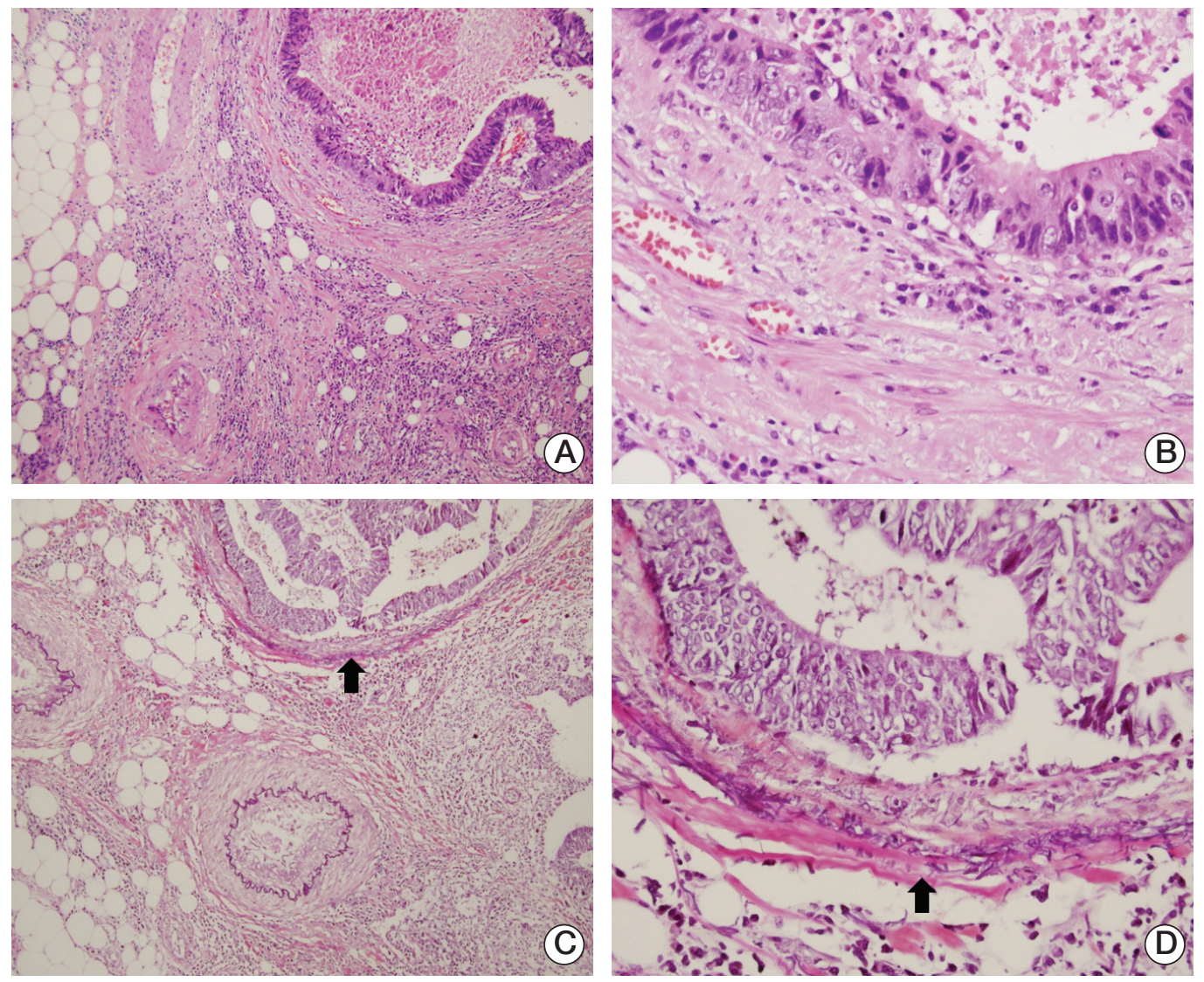

Fig. 3. (A) The tumor nodule with protruding tongue sign was identified (H\&E staining, $\times 100)$. (B) At high power, endothelial cells or the smooth muscular layer of a vessel around the tumor nodule were not identified (H\&E staining, $\times 400)$. (C, D) Elastic staining showed the thin elastic layer of a vessel around the tumor nodule with the protruding tongue sign (arrows) (C, elastic stain, $\times 100$, D, elastic stain, $\times 400)$.

data showed that detection of VI with morphologic features (protruding tongue and orphan arteriole signs) showed $77.8 \%$ sensitivity and $91.1 \%$ specificity with elastic stain as the gold standard and improved agreement between detection methods. These data indirectly suggest that use of morphologic findings (protruding tongue and orphan arteriole signs) is a comparable method and could enhance the detection rate of VI in CRC patients. However, $22.2 \%$ of cases (10/45 cases) showed false negative results (negative morphologic findings which showed positive on elastic stain), whereas $6.3 \%$ of cases ( $3 / 48$ cases) showed false-positive results (positive morphologic findings which showed negative on elastic stain). It is suggested that more studies regarding morphologic clues are needed to clarify these issues in daily surgical pathology practice of CRC resection specimens.

\section{Conclusion}

Taken together, the published body of work and our data recommend that pathologists should perform a careful review of H\&E slides in CRC cases with special attention to the protruding tongue and orphan arteriole signs in routine surgical practice and that elastic staining should be considered for equivocal cases. In addition, studies from Ontario, Canada suggest the benefits of national or multi-institutional educational programs (as knowledge transfer on VI detection at a national level) to improve VI detection rates and reduce interobserver variability in Korea. 
Table 2. Relationship between venous invasion identified by elastic stain and clinicopathological characteristics in 93 patients with T3 or T4 colorectal cancer

\begin{tabular}{|c|c|c|c|c|}
\hline \multirow{2}{*}{ Variable } & \multirow{2}{*}{ No. } & \multicolumn{2}{|c|}{ Venous invasion } & \multirow{2}{*}{ p-value } \\
\hline & & Absent & Present & \\
\hline Age (yr) & 93 & $68.4 \pm 1.54$ & $66.6 \pm 1.69$ & 0.422 \\
\hline Size $(\mathrm{cm})$ & 93 & $6.13 \pm 0.39$ & $5.40 \pm 0.26$ & 0.130 \\
\hline \multicolumn{5}{|l|}{ Sex } \\
\hline Male & 52 & $29(55.8)$ & $23(44.2)$ & 0.366 \\
\hline Female & 41 & $19(46.3)$ & $22(53.7)$ & \\
\hline \multicolumn{5}{|l|}{ Location } \\
\hline Right colon & 26 & $16(61.5)$ & $10(38.5)$ & 0.233 \\
\hline Left colon & 67 & $32(47.8)$ & $35(52.2)$ & \\
\hline \multicolumn{5}{|l|}{ Histological type } \\
\hline Well & 2 & $2(100)$ & 0 & 0.189 \\
\hline Moderately & 74 & $37(50.0)$ & $37(50.0)$ & \\
\hline Poorly & 10 & $3(30.0)$ & $7(70.0)$ & \\
\hline Mucinous & 7 & $6(76.9)$ & $1(14.3)$ & \\
\hline \multicolumn{5}{|l|}{ Perineural invasion } \\
\hline Negative & 45 & $29(64.4)$ & $16(35.6)$ & 0.017 \\
\hline Positive & 48 & $19(39.6)$ & $29(60.4)$ & \\
\hline \multicolumn{5}{|c|}{ Lymphovascular emboli } \\
\hline Negative & 75 & $47(62.7)$ & $28(37.3)$ & $<0.001$ \\
\hline Positive & 18 & $1(5.6)$ & $17(94.4)$ & \\
\hline \multicolumn{5}{|c|}{ Lymph node metastasis } \\
\hline N0 & 43 & $32(74.4)$ & $11(25.6)$ & $<0.001$ \\
\hline N1 & 26 & $11(42.3)$ & $15(57.7)$ & \\
\hline N2 & 24 & $5(20.8)$ & $19(79.2)$ & \\
\hline \multicolumn{5}{|l|}{ Depth of invasion } \\
\hline T3 & 71 & $38(53.5)$ & $33(46.5)$ & 0.508 \\
\hline $\mathrm{T} 4$ & 22 & $10(45.5)$ & $12(54.5)$ & \\
\hline \multicolumn{5}{|l|}{ Growth pattern } \\
\hline Expanding+mixed & 80 & $42(52.5)$ & $38(47.5)$ & 0.671 \\
\hline Infiltrative & 13 & $6(46.2)$ & $7(53.8)$ & \\
\hline
\end{tabular}

Values are presented as mean \pm standard deviation or number $(\%)$.

\section{Conflicts of Interest}

Conflict of interest relevant to this article was not reported.

\section{Acknowledgments}

This study was supported by a grant (0920050) from the National R\&D Program for Cancer Control, Ministry for Health, Welfare and Family Affairs, Republic of Korea.

\section{References}

1. Washington MK, Berlin J, Branton P, Burgart LJ, Carter DK, Fitzgibbons PL, et al. Protocol for the examination of specimens from patients with primary carcinoma of the colon and rectum. Arch Pathol Lab Med. 2009;133:1539-51.
2. Chang HJ, Park CK, Kim WH, Kim YB, Kim YW, Kim HG, et al. A standardized pathology report for colorectal cancer. Korean J Pathol. 2006;40:193-203.

3. Chapuis PH, Dent OF, Fisher R, Newland RC, Pheils MT, 
Smyth E, et al. A multivariate analysis of clinical and pathological variables in prognosis after resection of large bowel cancer. Br J Surg. 1985;72:698-702.

4. Harrison JC, Dean PJ, el-Zeky F, Vander Zwaag R. From Dukes through Jass: pathological prognostic indicators in rectal cancer. Hum Pathol. 1994;25:498-505.

5. Knudsen JB, Nilsson T, Sprechler M, Johansen A, Christensen $\mathrm{N}$. Venous and nerve invasion as prognostic factors in postoperative survival of patients with resectable cancer of the rectum. Dis Colon Rectum. 1983;26:613-7.

6. Betge J, Pollheimer MJ, Lindtner RA, Kornprat P, Schlemmer $\mathrm{A}$, Rehak $\mathrm{P}$, et al. Intramural and extramural vascular invasion in colorectal cancer: prognostic significance and quality of pathology reporting. Cancer. 2012;118:628-38.

7. Merkel S, Wein A, Gunther K, Papadopoulos T, Hohenberger W, Hermanek P. High-risk groups of patients with Stage II colon carcinoma. Cancer. 2001;92:1435-43.

8. Krasna MJ, Flancbaum L, Cody RP, Shneibaum S, Ben Ari G. Vascular and neural invasion in colorectal carcinoma. Incidence and prognostic significance. Cancer. 1988;61:1018-23.

9. Abdulkader M, Abdulla K, Rakha E, Kaye P. Routine elastic staining assists detection of vascular invasion in colorectal cancer. Histopathology. 2006;49:487-92.

10. Dirschmid K, Lang A, Mathis G, Haid A, Hansen M. Incidence of extramural venous invasion in colorectal carcinoma: findings with a new technique. Hum Pathol. 1996;27:1227-30.

11. Messenger DE, Driman DK, McLeod RS, Riddell RH, Kirsch R. Current practice patterns among pathologists in the assessment of venous invasion in colorectal cancer. J Clin Pathol. 2011;64:983-9.

12. Morris M, Platell C, de Boer B, McCaul K, Iacopetta B. Population-based study of prognostic factors in stage II colonic cancer. Br J Surg. 2006;93:866-71.

13. Maughan NJ, Morris E, Forman D, Quirke P. The validity of the Royal College of Pathologists' colorectal cancer minimum dataset within a population. Br J Cancer. 2007;97:1393-8.
14. Kirsch R, Messenger DE, Riddell RH, Pollett A, Cook M, Al-Haddad S, et al. Venous invasion in colorectal cancer: impact of an elastin stain on detection and interobserver agreement among gastrointestinal and nongastrointestinal pathologists. Am J Surg Pathol. 2013;37:200-10.

15. Howlett CJ, Tweedie EJ, Driman DK. Use of an elastic stain to show venous invasion in colorectal carcinoma: a simple technique for detection of an important prognostic factor. J Clin Pathol. 2009;62:1021-5.

16. Loughrey MB, Quirke P, Shepherd NA. Dataset for colorectal cancer histopathology reports [Internet]. London: The Royal College of Pathologists; 2016 [cited 2016 Feb 5]. Available from: https: //www.rcpath.org/ resourceLibrary/dataset-forcolorectal-cancer-histopathology-reports--3rd-edition-.html.

17. Stephen A, Brown I, Ellis D, Hawkins N, Hicks S, Hunter A, et al. Colorectal cancer structured reporting protocol [Internet]. Surry Hills: The Royal College of Pathologists of Australasia; 2012 [cited 2016 Feb 2]. Available from: http://www. rcpa.edu.au/getattachment / 95109a49-9e15-4038-9858cf314d9c7422/ Protocol-colorectal-cancer.aspx.

18. Kojima M, Shimazaki H, Iwaya K, Kage M, Akiba J, Ohkura $\mathrm{Y}$, et al. Pathological diagnostic criterion of blood and lymphatic vessel invasion in colorectal cancer: a framework for developing an objective pathological diagnostic system using the Delphi method, from the Pathology Working Group of the Japanese Society for Cancer of the Colon and Rectum. J Clin Pathol. 2013;66:551-8.

19. Messenger DE, Driman DK, Kirsch R. Developments in the assessment of venous invasion in colorectal cancer: implications for future practice and patient outcome. Hum Pathol. 2012;43:965-73.

20. Dawson H, Kirsch R, Driman DK, Messenger DE, Assarzadegan N, Riddell RH. Optimizing the detection of venous invasion in colorectal cancer: the ontario, Canada, experience and beyond. Front Oncol. 2014;4:354. 